\title{
Caracterização epidemiológica das mortes por doença de Chagas ocorridas no Brasil
} no período de 2010 a 2019

\author{
Epidemiological characterization of mortality from Chagas disease in Brazill from 2010 to 2019
}

Caracterización epidemiológica de la por enfermedad de Chagas en Brasil de 2010 a 2019

William Gonçalves

ORCID: https://orcid.org/0000-0003-1696-7024 Universidade Estadual de Alagoas, Brasil E-mail: gonwill29@gmail.com

Dalton Ferreira Matos

ORCID: https://orcid.org/0000-0002-6188-7536 Universidade Federal de Sergipe, Brasi E-mail: daltonmatosgn@gmail.com

Wandklebson Silva da Paz

ORCID: https://orcid.org/0000-0002-2898-2646

Universidade Federal de Pernambuco, Brasil

E-mail: wandklebson.paz@gmail.com

Juliana Vieira de Souza

ORCID: https://orcid.org/0000-0001-6397-5049 Instituto Federal do Pará, Brasil E-mail: vieirajuliana510@gmail.com

Allan Bruno Alves de Sousa Santos ORCID: https://orcid.org/0000-0001-6412-7164

Faculdade de Educação São Francisco, Brasil E-mail: Abass@faesf.com.br

Maria Gisely Martins dos Santos ORCID: https://orcid.org/0000-0003-1905-9371

Universidade Estadual de Alagoas, Brasil

E-mail: giselymartins21quimica@ gmail.com

Samuel Felício de Oliveira

ORCID: https://orcid.org/0000-0001-6482-4808

Centro Universitário Alfredo Nasser, Brasil

E-mail: felicio.samuel306@gmail.com

Mikaelle Alves Silva

ORCID: https://orcid.org/0000-0002-9336-1491

Universidade Federal do Espírito Santo, Brasil

E-mail: farm.mikaelle@gmail.com

Mayane Luiza Alves Nunes

ORCID: https://orcid.org/0000-0002-2646-8707

Universidade Estadual de Alagoas, Brasil

E-mail: maayanenunes@gmail.com

Robert Lincoln Barros Melo

ORCID: https://orcid.org/0000-0002-3430-4411

Fundação Oswaldo Cruz, Brasil

E-mail: robert.lincoln.b@gmail.com

Marcelo Felix Feitosa Ricardo

ORCID: https://orcid.org/0000-0002-4655-301X

Universidade Estadual de Alagoas, Brasil

E-mail: marcelofelixfeitosaricardo@gmail.com

Jhonatan Ferreira da Silva

ORCID: https://orcid.org/0000-0003-2292-2172

Universidade Estadual de Alagoas, Brasi

E-mail: jhonatanferreira615@ gmail.com

Leticia Pereira Bezerra

ORCID: https://orcid.org/0000-0001-6375-633X Universidade Federal de Sergipe, Brasil

E-mail: pleticia706@gmail.com

Márcio Thomaz dos Santos Varjão ORCID: https://orcid.org/0000-0002-9916-1490

Universidade Estadual de Alagoas, Brasil E-mail: marciot20@hotmail.com 


\title{
Resumo
}

A doença de Chagas ou Tripanossomíase americana é a infecção causada pelo protozoário Trypanosoma cruzi transmitido por um inseto triatomíneo. Em indivíduos infectados, essa enfermidade pode comprometer o coração ou trato gastrointestinal sendo potencialmente fatal. Estima-se que aproximadamente 6 a 7 milhões de pessoas estejam infectadas com T. cruzi em todo o mundo. A doença de Chagas representa um desafio principalmente para a saúde pública da América Latina, onde ela é endêmica em 21 países, incluindo o Brasil. Diante disso, a presente pesquisa tem como objetivo verificar a mortalidade por Doença de Chagas no Brasil durante os anos de 2010 a 2019, por meio de um estudo descritivo transversal, empregando dados de base populacional do Sistema de Informação sobre Mortalidade (SIM) do Ministério da Saúde brasileiro. Durante a análise verificou-se que no período avaliado foram registados no SIM, 45.409 óbitos nas cinco regiões brasileiras. Em relação aos casos relatados, as características sociodemográficas predominantes foram: sexo masculino (54,38\%), faixa etária entre 70 a 79 anos $(28,02 \%)$, raça/cor pardas $(42,09 \%)$, e escolaridade $<8$ anos $(45,47 \%)$. Os resultados obtidos indicam uma relação entre a vulnerabilidade social e o acometimento das doenças de Chagas. Além disso, ficou evidente que, apesar dos avanços, o número de óbitos registrados no Brasil permanece em um patamar bastante elevado. O SIM demonstrou ser um importante instrumento para realização de estudos epidemiológicos, entretanto, foram observadas algumas limitações quanto a qualidades dos registos, evidenciando algumas falhas.

Palavras-chave: Tripanossomíase americana; Doença de Chagas; Doenças tropicais negligenciadas.

\begin{abstract}
Chagas disease or American trypanosomiasis is an infection caused by the protozoan Trypanosoma cruzi transmitted by a triatomine insect. In infected individuals, this disease can compromise the heart or gastrointestinal tract and is potentially fatal. It is estimated that approximately 6 to 7 million people are infected with T. cruzi worldwide. Chagas disease represents a challenge mainly for public health in Latin America, where it is endemic in 21 countries, including Brazil. Therefore, this research aims to verify the mortality from Chagas Disease in Brazil during the years 2010 to 2019, through a cross-sectional descriptive study, using population-based data from the Mortality Information System (SIM) of the Ministry of Brazilian Health. During the analysis, it was found that in the period evaluated, 45,409 deaths were registered in the SIM in the five Brazilian regions. Regarding the reported cases, the predominant sociodemographic characteristics were: male (54.38\%), aged between 70 and 79 years (28.02\%), brown race/color $(42.09 \%)$, and education $<8$ years $(45.47 \%)$. The results obtained indicate a relation between social vulnerability and the involvement of Chagas disease. In addition, it was evident that, despite advances, the number of deaths registered in Brazil remains at a very high level. The SIM proved to be an important tool for conducting epidemiological studies, however, some limitations were observed regarding the quality of the records, evidencing some flaws.
\end{abstract}

Keywords: American trypanosomiasis; Chagas Disease; Neglected tropical diseases.

\section{Resumen}

La enfermedad de Chagas o tripanosomiasis americana es la infección causada por el protozoo Trypanosoma cruzi transmitida por un insecto triatomino. En individuos infectados, esta enfermedad puede comprometer el corazón o el tracto gastrointestinal siendo potencialmente fatal. Se estima que aproximadamente 6 a 7 millones de personas están infectadas con $T$. cruzi en todo el mundo. La enfermedad de Chagas representa un desafío principalmente para la salud pública en América Latina, donde es endémica en 21 países, incluido Brasil. Por lo tanto, esta investigación tiene como objetivo verificar la mortalidad por enfermedad de Chagas en Brasil durante los años 2010 a 2019, a través de un estudio descriptivo transversal, utilizando datos de base poblacional del Sistema de Información de Mortalidad (SIM) del Ministerio de la Salud de Brasil. Durante el análisis, se registraron 45.409 muertes en el período SIM en las cinco regiones brasileñas. En cuanto a los casos relatados, las características sociodemográficas predominantes fueron: sexo masculino (54,38\%), grupo e edad entre 70 y 79 años $(28,02 \%)$, mestizo/color $(42,09 \%)$, y $<8$ años $(45,47 \%)$. Los resultados obtenidos indican una relación entre la vulnerabilidad social y la afectación de las 
enfermedades de Chagas. Además, fue evidente que, a pesar de los avances, el número de muertes registradas en Brasil se mantiene en un nivel muy alto. SIM demostró ser un instrumento importante para la realización de estudios epidemiológicos, sin embargo, se observaron algunas limitaciones con respecto a las cualidades de los registros, evidenciando algunos defectos.

Palabras clave: Tripanosomiasis americana; Enfermedad de Chagas; Enfermedades tropicales desatendidas.

\section{Introdução}

A doença de Chagas ou tripanossomíase mericana é a infecção causada pelo protozoário Trypanosoma cruzi. A principal forma de transmissão é a vetorial, por meio de triatomíneos, conhecido popularmente como barbeiro. O $T$. cruzi possui diversos hospedeiros. Esse protozoário tem a capacidade de infectar diversas espécies de mamíferos, como por exemplo guaxinins, gambás, ratazanas, cães e seres humanos (Bern et al., 2019). Estima-se que aproximadamente 6 a 7 milhões de pessoas estejam infectadas com T. cruzi em todo o mundo. A doença de Chagas representa um desafio principalmente para a saúde pública da América Latina, onde ela é endêmica em 21 países, incluindo o Brasil (WHO, 2021). Atualmente, essa enfermidade é uma das principais doenças Tropicais Negligenciadas (DTN) (Echeverría et al., 2021) e apesar dos esforços de vários pesquisadores, o número de mortes de indivíduos chagásicos ainda é preocupante (Cordeiro et al., 2021).

A soroprevalência dessa doença é maior na população rural, porém o perfil de soropositividade tanto na população rural quanto urbana há uma maior prevalência de soropositividade nos grupos etários, com faixas etárias mais velhas (Echalar et al., 2021). Segundo o boletim epidemiológico do Ministério da saúde (2020), no período de 2007 a 2016 , a doença de Chagas acometeu cerca de 850 pessoas com idade média de 33 anos, tendo maior prevalência de casos notificados em os indivíduos do sexo masculino (54,40\%). Em relação ao número de casos entre mulheres, a faixa de idade reprodutiva gera maior preocupações devido a possibilidade de transmissão congênita (Ikedionwu et al., 2020).

A forma de tratamento para essa doença consiste basicamente com o uso do Benzonidazol, onde esse medicamento não possui evidencias concretas a respeito da fase crônica na doença de Chagas (Brito et al., 2021). O Sistema Único de Saúde do Brasil (SUS), através dos seus valores e princípios principais, como universalidade, equidade, integralidade, devem ser os pilares de qualquer política e dos programas de interesse público. Pois no Brasil os movimentos sociais voltados para saúde devem ser valorizados e estimulados, pois deve-se buscar ações para o enfrentamento da doença de Chagas no país (Dias et al., 2016).

Outra questão que deve ser considerada é o incentivo da pesquisa no país, pois nos últimos anos o número de trabalhos voltados para essa doença vem diminuindo com o passar dos anos como foi retratado por Lima et al, (2020) onde nos anos de 2014 a 2018 tiveram um total de 14 publicações, sendo que em 2017 foi o ano que apresentou o menor número com um total de 2 publicações, com isso mostra que essa doença é negligenciada pelos governantes, não só nos investimentos na saúde pública, mas também no incentivo na pesquisa cientifica (Silva et al., 2021).

Com isso, verificar dados sociodemográficos a respeito da mortalidade de pessoas no Brasil pode favorecer uma compreensão dos dados a respeito de uma determinada população, favorecendo a construção de melhores políticas públicas a respeito da saúde dessas pessoas, aumentando também o número do acervo científico em pesquisas voltadas para essa doença. Por conseguinte, o objetivo deste estudo é relatar o número de mortes ocorridos por doença de Chagas no Brasil durante os anos de 2010 a 2019, empregando dados de base populacional, de acordo com Sistema de Informações sobre Mortalidade (SIM). 


\section{Metodologia}

\subsection{Tipo e Área de Estudo}

Conduzimos um estudo descritivo transversal, com o intuito de verificar as mortes relacionadas a doença de Chagas ocorridas no Brasil entre 2010 a 2019. O país possui 27 unidades federativas, com uma área territorial de 8.510.345,538 km². A população do país se estima em 211.755 .692 habitantes e possui densidade demográfica de 22,43 habitantes/km² (IBGE, 2020).

\subsection{Fonte de Dados}

Os dados sobre os óbitos da doença foram obtidos do Departamento de Informática do Sistema Único de Saúde (DATASUS) da Secretaria de Vigilância em Saúde do Ministério da Saúde (SVS/MS) a partir dos bancos de dados do Sistema de Informação sobre Mortalidade. Esses dados são de domínio público, dessa forma, não houve necessidade de apreciação ética nem de autorização de acesso aos dados.

\subsection{Procedimentos Metodológicos}

Primeiramente, foram selecionadas todas as mortes por doença de Chagas ocorridas por local de residência no Brasil, entre os anos de 2010 e 2019. A seleção dos dados ocorreu independentemente da forma clínica da doença ou da sua menção como causa básica ou associada. A partir da seleção das mortes, foi feita a categorização e quantificação das mortes de acordo com as variáveis sociodemográficas disponíveis no SIM, sendo elas: região brasileira, sexo, faixa etária, raça/cor e escolaridade. As mortes também foram categorizadas de acordo com o ano de ocorrência no Brasil. Os dados foram armazenados através do software Microsoft Word Excel 2019, onde foram elaborados as tabelas e gráficos deste trabalho.

\section{Resultados e Discussão}

Em relação as características sociodemográficas predominantes dos casos foram: sexo masculino com $(54,38 \%)$, faixa etária em idade igual ou superior que 60 anos (74,99\%), pessoas pardas com 19.113 (42,09\%), e pessoas com menos de 8 anos de escolaridade $(45,47 \%)$. No entanto em relação a questão da escolaridade esse número pode ter sido maior, pois cerca de $(23,48 \%)$ dos dados foram ignorados e/ou notificados (Tabela 1$)$.

O maior número de casos no sexo masculino é frequentemente relatado na literatura, pois esse grupo tem maior risco de exposição ao parasito devido às atividades laborais (Ledezma et Al., 2020; Amorim; Costa, 2021). No entanto, a doença de Chagas no país é considerada uma das enfermidades negligenciadas mais importantes, demonstrando uma variação regional que tem implicações sociais e de saúde para as pessoas (Martins-Melo et al., 2021).

O exacerbado número de casos em indivíduos de faixa etária igual ou superior a 60 anos, pode estar relacionado por se tratar de uma idade avançada, que consequentemente contribui para o agravo da doença e evolução de quadros clínicos da mesma. Onde a capacitação dos profissionais em enfermagem é essencial para que consigam identificar os sintomas da doença, fazendo com que a doença de Chagas não evolua (Freitas et al., 2019; Porfírio et al., 2020).

Sendo considerada mais grave e potencialmente letal em idosos, faz-se necessário que o estado deva intervir e dar assistência médica a esse grupo etário, pois a doença de Chagas é um problema de saúde individual e populacional para o idoso (Lima-Costa et al., 2010). 
Tabela 1. Características sociodemográficas da população com doença de Chagas no Brasil, 2010 a 2019.

\begin{tabular}{lll}
\hline Variáveis & $\mathbf{N}^{\mathbf{0}}$ absoluto de casos & Frequência $(\%)$ \\
\hline Gênero & & \\
Masculino & 24.694 & 54,38 \\
Feminino & 20.711 & 45,60 \\
Ignorado/Branco & 4 & 0,02
\end{tabular}

\section{Faixa etária}

\begin{tabular}{|c|c|c|}
\hline$\leq 1$ a 9 anos & 11 & 0,02 \\
\hline $10-19$ & 35 & 0,08 \\
\hline $20-29$ & 179 & 0,39 \\
\hline $30-39$ & 1.080 & 2,38 \\
\hline $40-49$ & 3.183 & 7,01 \\
\hline $50-59$ & 6.863 & 15,11 \\
\hline$\geq 60$ & 34.051 & 74,99 \\
\hline Ignorado/Branco & 7 & 0,02 \\
\hline Raça/Cor & & \\
\hline Branco & 17.956 & 39,54 \\
\hline Preto & 5.691 & 12,53 \\
\hline Amarelo & 156 & 0,34 \\
\hline Parda & 19.113 & 42,09 \\
\hline Indígena & 57 & 0,12 \\
\hline Ignorado/Branco & 2.436 & 5,36 \\
\hline Escolaridade & & \\
\hline Analfabeto & 11.009 & 24,24 \\
\hline$<8$ anos & 20.650 & 45,47 \\
\hline$\geq 8$ anos & 3.086 & 6,79 \\
\hline Ignorado/Branco & 10.664 & 23,48 \\
\hline
\end{tabular}

Fonte: Autores.

Entre os anos de 2010 a 2019, foram registrados um total de 45.409 óbitos nas cinco regiões brasileiras. Dessas cinco regiões, a Sudeste foi a que teve o maior destaque no número de óbitos por Doença de Chagas, apresentando 21.409 (47,14\%) óbitos registrados. Em contrapartida, a região que obteve o menor índice foi a região Norte com 872 (1,92\%) óbitos notificados Tabela 2. 
De acordo com os nossos dados, foi possível analisar que houve uma diminuição na taxa de mortalidade nas regiões brasileiras durante o período de estudo, ou seja, o número de mortes por doença de Chagas diminuiu com o passar dos anos. Em 2010 o número de mortes era 4.876 (10,73\%) e em 2019 esse número diminuiu para 4.287 (9,44\%) Figura 1. No entanto, é necessário levar em consideração que, por se tratar de dados secundários de uma única plataforma, pode existir também falhas nas notificações e registros de agravos, em que estas informações podem apresentar limitações como já foram relatadas por outros pesquisadores em diversos estudo (Confortin et al., 2019; Silva et al., 2021).

Figura 1. Número de óbitos por doença de Chagas durante os anos de 2010 a 2019 no Brasil.

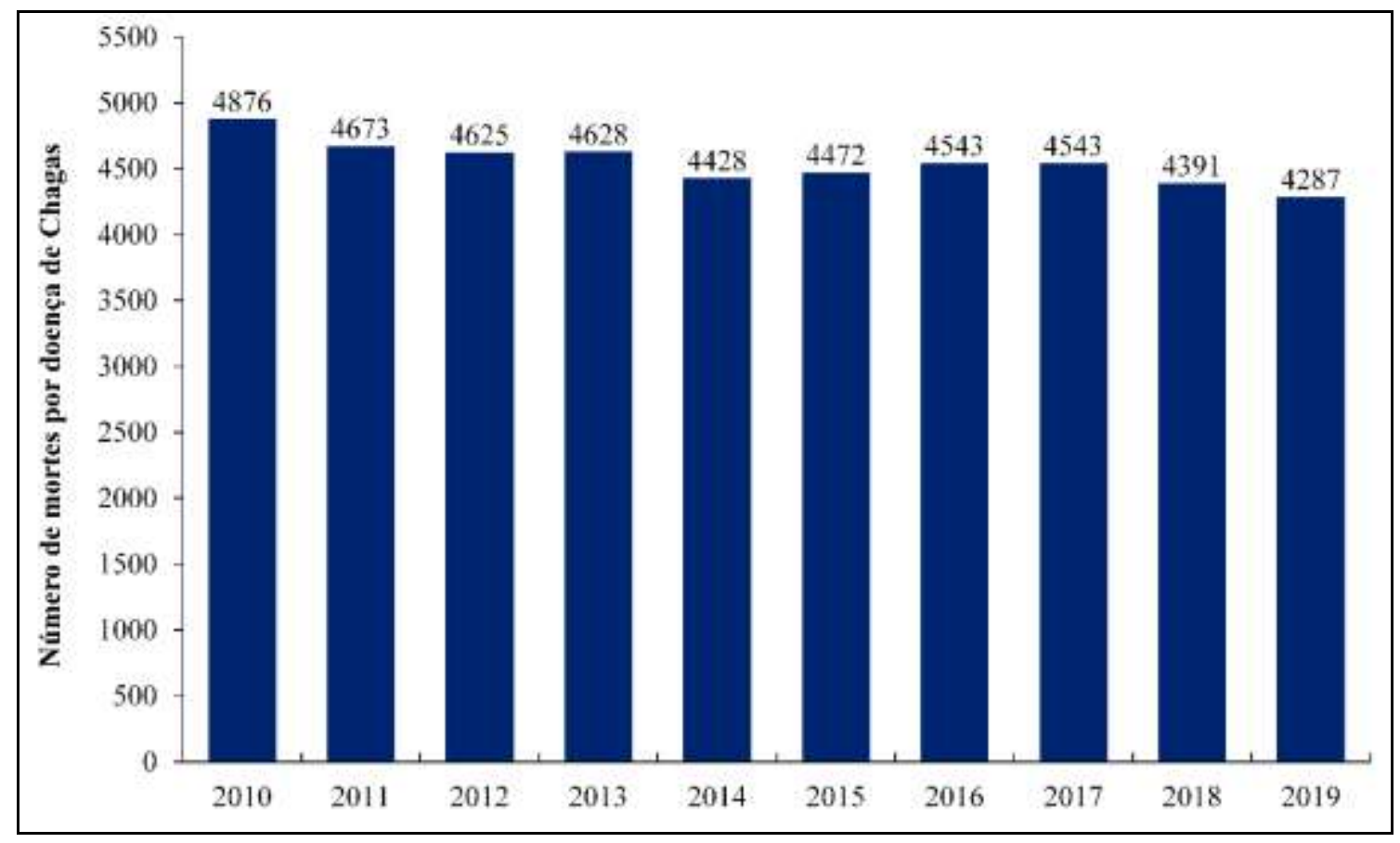

Fonte: Autores.

$\mathrm{Na}$ literatura cientifica existem diversas pesquisas no âmbito de mortalidade por agravamentos de doença de chagas em regiões, ou até mesmo em estados brasileiros, como é o caso do trabalho de Amorim e Costa (2021), onde os pesquisadores verificaram a tendencia temporal dos anos 2008 a 2018 na Bahia, em que os dados obtidos demonstraram nesse estudo que no ano de 2008 o estado baiano teve 646 óbitos notificados e em 2018 houve uma queda na qual foi registrado 613 óbitos, tendo em vista que nossos dados mostraram que o estado da Bahia foi o estado com o maior número de mortes nos últimos 9 anos na região nordeste, com 6.142 óbitos $(13,52 \%)$.

Em se tratando da região Centro-oeste, Felipe et al. (2020), mostram que entre os anos de 2008 a 2017, a mesma teve um total de 5.663 óbitos por decorrência da doença de chagas no período de estudo avaliado, onde Goiás foi o estado com o maior índice de óbitos, corroborando com os nossos dados, que demonstraram que este estado registrou 6.907 óbitos últimos 9 anos.

Em um estudo proposto por Silva, Junior e Dantas (2019), foi descrito o perfil de morbidade no Nordeste brasileiro por doença de chagas, onde os autores verificaram que no ano de 2008 teve um total 374 internações e 18 óbitos, já em 2018 teve um total de 222 internações e 18 óbitos por doença de Chagas.

Com isso, o tratamento desses pacientes chagásicos se dá de acordo com a gravidade da doença, colaborando para o risco dos efeitos colaterais dos medicamentos existentes atualmente no mercado, necessitando de uma droga farmacológica que não cause efeitos colaterais drásticos nesses pacientes (Barreto et al., 2021). Porém, não existe nenhuma vacina disponibilizada 
no mercado que seja eficaz contra essa doença, no entanto, existe vacina em fase pré-clínica contra o Trypanosoma cruzi, podendo está no mercado em um futuro próximo (Luna; Campos, 2020).

Em se tratando da região Norte do país, nos últimos 9 anos foram notificados um total de 872 (23,78\%) óbitos, resultados estes que evidenciam similaridade com outros trabalhos na literatura cientifica, mostrando que essa região foi a que possuiu o menor número de óbitos no país, porém essas informações devem ser interpretadas com cautela (Martins-Melo et al., 2019; Souza et al., 2021). Pois os dados epidemiológicos determinam uma pré-disposição de se aumentar na região Norte, necessitando de uma implementação dos indicadores ambientais, não só para essa região com o intuito da prevenção precoce dos agravos dessa doença (Costa et al., 2018). Com isso é necessária uma melhor compreensão do perfil epidemiológico dessa doença, para poder assim promover o controle dos possíveis focos de disseminação desse protozoário.

Tabela 2. Taxa de mortalidade da doença de Chagas em regiões do Brasil, durante os anos de 2010 a 2019

\begin{tabular}{lll}
\hline Regiões Brasileiras & $\mathbf{N}^{\mathbf{0}}$ absoluto de casos & Frequência (\%) \\
\hline Região Norte & 872 & 1,92 \\
Região Nordeste & 10.159 & 22,37 \\
Região Sudeste & 21.409 & 47,14 \\
Região Sul & 2.167 & 4,77 \\
Região Centro-Oeste & 10.802 & 23,78 \\
Total & $\mathbf{4 5 . 4 0 9}$ & $\mathbf{1 0 0}$ \\
\hline
\end{tabular}

Fonte: Autores.

Por meio dos dados apresentados no presente estudo, é importante considerar que este trabalho apresenta algumas limitações resultantes do Sistema de Informação sobre Mortalidade (SIM) que, por sua vez, é o único sistema oficial do Brasil e pode apresentar inconsistência na disposição e qualidade dos dados, onde consequentemente, pode afetar na análise e avaliação por se tratar de um trabalho de utilização de dados secundários. No entanto, apesar das limitações mencionadas, os resultados do presente estudo mostram coerência e credibilidade com conhecimentos existentes sobre a doença de Chagas.

\section{Conclusão}

Diante desse estudo, foi verificado que os maiores percentuais, em relação às variáveis sociodemográficas, concentraram-se no sexo masculino, faixa etária de 20 a 39 anos, pessoas com cor de pele não branca, baixa escolaridade. Ressalta-se, ainda, que houve alto percentual de casos ignorados e/ou em branco, podendo esses dados serem subestimados. Com isso o estado deve intervir através de investimentos não só na saúde pública, mas também investimentos na ciência, pois nos últimos anos o número de investimentos em relação à pesquisa no Brasil vem sofrendo cortes drásticos, afetando a qualidade da ciência no país.

É imprescindível salientar que esse estudo apresentou limitações referentes ao Sistema de Informação sobre Mortalidade (SIM), em que o sistema não notifica dados a respeito de pacientes chagásicos na fase aguda e crônica, dificultando a apuração e tabulação de dados. 


\section{Referências}

Amorim, D. S. \& Costa, M. S. F. (2021) Tendência da mortalidade por doença de Chagas na Bahia: Entre os anos de 2008 a 2018. Research, Society and Development, $10(5)$.

Barreto., P. G. S et al (2021) Descrição de eventos adversos a medicamentos em pacientes hospitalizados com a doença de Chagas. Revista Multidisciplinar Em Saúde, 2 (1), 58.

Bern, C et al (2019) Chagas disease in the United States: a public health approach. Clinical microbiology reviews, 33 (1).

Brito, A. C. O et al (2021) Um Doenças Negligenciadas: Doença de Chagas e os aspectos atuais do tratamento. Revista de Ensino, Ciência e Inovação em Saúde, 2 (1), 9-17.

Costa, M. M. R et al (2018) Doença de chagas: tendência epidemiológica por regiões do Brasil. Brazilian Journal of Health Review, 1 (1), $252-259$.

Cordeiro, T. A. R et al (2021) Electrochemical biosensors for neglected tropical diseases: A review. Talanta.

Confortin, S. C et al (2019) Mortalidade prematura pelas principais doenças crônicas não transmissíveis nos estados do Brasil. Revista Brasileira de Enfermagem, 72, 1588-1594.

Dias, J. C. P et al (2016) II Consenso Brasileiro em Doença de Chagas, 2015. Epidemiol. Serv. Saúde, Brasília, 25, 7-86.

Echalar, J-C et al (2021) Age-related anomalies of electrocardiograms in patients from areas with differential Seroprevalence of Chagas disease in Southern Bolivia." Parasite Epidemiology and Control. 13, e00204.

Echeverría, L. E et al (2021) Coagulation disorders in Chagas disease: A pathophysiological systematic review and meta-analysis. Thrombosis Research, 201, 73-83.

Felipe, A. G. B et al (2020) Doença de chagas: Análise de mortalidade na região Centro-Oeste do Brasil (2008 a 2017). Brazilian Journal of Health Review, v. $3(6), 16102-16107$.

Franco-Paredes, C et al (2020) A deadly feast: Elucidating the burden of orally acquired acute Chagas disease in Latin America-Public health and travel medicine importance. Travel medicine and infectious disease.

Freitas, M. J. C et al (2019) Cardiopatia chagásica em pacientes idosos. Brazilian Journal of Health Review, 2 (4), $3283-3285$.

IBGE. Instituto Brasileiro de Geografia e Estatística. Brasil. 2020.: https://www.ibge.gov.br/cidades-e-estados.html.

Ikedionwu, C et al (2020) Trends and associated characteristics for Chagas disease among women of reproductive age in the United States, 2002 to 2017. Parasite Epidemiology and Control.

Ledezma, A. P et al (2020) Mixed infections by different Trypanosoma cruzi discrete typing units among Chagas disease patients in an endemic community in Panama. PloS one, 15 (11).

Lima-Costa, M. F et al (2010) Chagas disease and mortality in old age as an emerging issue: 10 year follow-up of the Bambuí population-based cohort study (Brazil). International Journal of Cardiology, 145, 362-363.

Lima, E. A. N et al (2020) T. A. Análise das produções científicas relacionadas à Doença de Chagas. Brazilian Archives of Health and Environment, 1 (1), 1624.

Luna, E. J. A. \& Campos, S. R. S. L. C. (2020) O desenvolvimento de vacinas contra as doenças tropicais negligenciadas. Cadernos de Saúde Pública.

Martins-Melo, F. R et al (2019) Burden of Chagas disease in Brazil, 1990-2016: findings from the Global Burden of Disease Study 2016. International journal for parasitology, 49 (3-4), 301-310.

Martins-Melo, F. R et al (2021) Levels and trends in Chagas disease-related mortality in Brazil, 2000-2019. Acta Tropica.

Ministério da Saúde (BR). Secretaria de Vigilância em Saúde. Doença de Chagas: 14 de abril -Dia Mundial. Bol Epidemiol. 2020. http://www.saude.gov.br/boletins-epidemiologicos.

Neves, D. P et al (2016) Parasitologia Humana. (13a ed.), Editora Atheneu.

Porfírio, D. M et al (2020) Prevalência de Doença de Chagas em Idosos no Estado do Pará: Uma Análise Retrospectiva. Brazilian Journal of Health Review, 3 (4), 9142-9152.

Silva, A. P et al (2019) Doença de Chagas: Perfil de morbidade hospitalar na Região do Nordeste Brasileiro. Revista de Ciências da Saúde Nova Esperança, 17 (3), 08-17.

Silva, P. L. N et al (2021) Impacto do déficit de investimentos para o tratamento da doença de chagas no Brasil: revisão narrativa. Nursing (São Paulo), 24 (275), 5514-5529.

Silva, M. S et al. Internações e mortalidade de cirurgias otorrinolaringológicas nos estados brasileiros no período de 2011 a 2021 . Research, Society and Development, 10 (8), e58910817678-e58910817678.

Souza, S. B et al (2021) Perfil epidemiológico da doença de Chagas aguda na região norte do Brasil no ano de 2015-2019. Revista Eletrônica Acervo Saúde,13 (7), 8200-8200.

WHO - WORLD HEALTH ORGANIZATION. Chagas disease - American trypanosomiasis (2021). Geneva: World Health Organization. https://www.who.int/health-topics/chagas-disease\#tab=tab_1, 